Nouvelles perspectives en sciences sociales

Revue internationale de systémique complexe et d'études relationnelles

\title{
Et si la coopération était un mythe? Un pilier des sciences sociales ébranlé par la simulation
}

\section{Jean-Louis Dessalles}

Volume 5, numéro 2, mai 2010

Sur le thème de la simulation

URI : https://id.erudit.org/iderudit/044077ar

DOI : https://doi.org/10.7202/044077ar

Aller au sommaire du numéro

Éditeur(s)

Prise de parole

ISSN

1712-8307 (imprimé)

1918-7475 (numérique)

Découvrir la revue

Citer cet article

Dessalles, J.-L. (2010). Et si la coopération était un mythe? Un pilier des sciences sociales ébranlé par la simulation. Nouvelles perspectives en sciences sociales, 5(2), 79-89. https://doi.org/10.7202/044077ar
Résumé de l'article

De nombreux auteurs voient dans la coopération le ciment des sociétés humaines. Plus que les autres animaux, les humains seraient capables de renoncer à des bénéfices immédiats pour en faire profiter autrui, dans l'espoir d'une réciprocité future. Or, cette hypothèse est mise à mal par la simulation : les agents qui ne coopèrent pas, ou qui coopèrent moins, en ressortent gagnants. J'explore une autre hypothèse, inspirée par la modélisation du langage. Les actes qui nous semblent coopératifs seraient en réalité des signaux. Les individus ne se montrent pas prosociaux par calcul, mais pour afficher des qualités qui se trouvent être essentielles, dans notre espèce, pour se faire accepter en tant qu'ami et ainsi constituer un réseau social. 


\title{
Et si la coopération était un mythe? Un pilier des sciences sociales ébranlé par la simulation
}

\author{
Jean-Louis Dessalles \\ Telecom Paristech et ILCAA, Tokyo University of Foreign Studies
}

Ta société humaine se distingue de manière éclatante des Lsociétés animales par le niveau de coopération atteint entre individus non apparentés. Un exemple : je me suis coupé, récemment, en essayant de replier un parapluie bon marché. Le sang qui coulait de mon doigt a attiré plusieurs personnes inconnues de moi, l'une munie d'un mouchoir, une autre d'un pansement déjà prêt à se coller sur mon entaille, une autre encore me demandant, sans doute dans sa langue maternelle, si j'allais bien. La scène se passe au Japon; l'une des sociétés les plus coopérantes, pensera-t-on sans doute. Le lendemain, il y eut un typhon. De nombreux vélos étaient tombés à terre, renversés par le vent. Parmi les centaines de passants, aucun ne songeait à les relever. Pourtant, si un passant sur cent avait pris trois secondes pour redresser ne serait-ce qu'un seul vélo, la situation, désolante pour le regard, serait rapidement redevenue normale. La coopération humaine, dira-t-on, n'est pas systématique. Elle est subtile, elle dépend du contexte, etc.

La coopération est l'un des piliers, voire un axiome, des sciences sociales. Elle seule permet à des individus non apparen- 
tés de vivre ensemble. Même la guerre, sorte d'autodestruction des sociétés, repose sur une coopération efficace. Et, pour prendre un exemple que je connais bien, le langage est présenté aux étudiants comme un cas emblématique de coopération, consistant en un échange d'informations. C'est pourtant à propos du langage que j'ai eu mes premiers doutes. J'ai alors tenté de m'attaquer au pilier, armé du canif des modélisateurs, la simulation.

\section{Simuler la coopération}

Les premières simulations de la coopération, issues des travaux de Robert Axelrod et William Hamilton ${ }^{1}$, ont révélé un désastre, même si la situation a rarement été présentée comme telle. Depuis, des centaines d'articles scientifiques ont été consacrés au problème de la stabilisation de la coopération, aboutissant à des modèles souvent compliqués et souvent critiqués. En quoi consiste le problème, tel qu'il a été révélé par Axelrod et Hamilton? Tout simplement dans le fait que dans un monde coopérant, il est avantageux de ne pas coopérer. Dès que l'on est en présence d'un effort collectif et que cet effort est coûteux, le gagnant est celui qui laisse travailler les autres (en commençant peut-être par travailler $1 \%$ de moins que les autres). Le cas emblématique est celui, bien connu, de la tragédie des biens communs, qui trouve une illustration dramatique dans le traitement actuel de la question du réchauffement planétaire. La coopération, reconnaissons-le, ne va pas de soi.

Avant de continuer, dissipons un possible malentendu. Il existe, bien entendu, des situations, comme la symbiose, où chacun tire un bénéfice plus grand de la coopération que de la non-coopération. Dans d'autres cas moins favorables, l'action unilatérale reste encore préférable à la non-action (c'est le problème connu sous le nom du snow-drift, la congère qui barre la route à deux automobilistes, la question étant de savoir s'ils vont

Robert Axelrod et William D. Hamilton, "The Evolution of Cooperation ", Science, vol. 211, no 4489, 1981, p. 1390-1396 [en ligne] http://www.cdnresearch.net/pubs/others/axelrod_ham_1981.pdf; Robert Axelrod, The Evolution of Cooperation, New York, Basics Books, 1984. 
tous deux pelleter la neige pour pouvoir rentrer chez eux). Ces situations sont moins intéressantes, du point de vue des sciences sociales, que celles, beaucoup plus fréquentes, dans lesquelles il vaut mieux ne rien faire plutôt que de se faire avoir.

Il existe des solutions faciles à ce problème. Par exemple, on peut instituer une autorité qui punira tous ceux qui ne coopèrent pas. Cela fonctionne à peu près pour l'impôt dans nos sociétés, mais cela n'explique pas le pansement que la dame japonaise a collé sur mon pouce. Et que gagnent les individus qui punissent, à leurs propres risques, ceux qui ne coopèrent pas? On se retrouve avec un problème de coopération d'ordre deux, où il est avantageux de laisser les autres punir, si bien que le système de punition disparaît au bout d'un moment. Et puis, souvenons-nous que la plupart des conduites collectives humaines se font au quotidien sans intervention d'aucune autorité, à commencer par les innombrables jeux qui se déroulent simultanément dans la cour d'une école.

Pour sortir du problème des biens collectifs, les chercheurs ont depuis longtemps imaginé que la coopération pouvait exister entre individus qui montrent leur volonté de coopérer. Avant de s'engager dans l'action, les agents regardent qui sont les autres acteurs. S’ils disposent d'une mémoire et se souviennent des actions passées des uns et des autres, ils peuvent discriminer leurs partenaires. Une telle stratégie semble pouvoir exclure les tricheurs. Ceux-ci sont systématiquement mis à l'écart et ne tirent jamais profit d'une action collective aux dépens des autres. La coopération doit finalement l'emporter. Le raisonnement est limpide, implacable. Les simulations d'Axelrod ont pourtant ruiné cet espoir.

Axelrod s'est limité au cas de situations dyadiques. À chaque étape de ses simulations, deux joueurs pris dans une population d'agents sont amenés à décider s'ils entrent dans une action conjointe (par exemple, un échange de services : je répare ta voiture, tu fais ma comptabilité). Ils gagnent plus à coopérer qu’à ne pas coopérer, mais ils gagnent encore plus à ne pas coopérer 
si l'autre coopère. Mais si tu ne coopères pas, je saurai m'en souvenir.

Contre toute attente, la stratégie à mémoire la plus simple l'a emporté sur toutes les autres. Tit-for-tat (Tft: donnant-donnant) commence par coopérer, puis copie le comportement que l'autre a adopté précédemment. Tft est donc gentille avec ceux qui coopèrent; elle est méchante avec ceux qui trichent, mais elle est toujours prête à pardonner. Personne n'avait prévu le succès de Tft face à d'autres stratégies bien plus élaborées. Dans l'écologie des différentes stratégies, Tft s'en sort le mieux en moyenne.

Un monde entièrement $T f t$ peut-il offrir une image intéressante des sociétés humaines? Non. Déjà, un monde Tft pur est instable. Des tricheurs qui ne coopèrent jamais peuvent l'envahir, car ils exploitent facilement toutes les $T f t$ qui ne les connaissent pas. Un tel monde se met à osciller entre des phases plutôt coopératives et des phases de chacun-pour-soi ${ }^{2}$. Et puis, la plupart des conduites collectives humaines impliquent plus de deux acteurs. Dans ces conditions non dyadiques, une stratégie du type $T f t$ aura toujours une raison de ne pas coopérer. Enfin, je ne crois pas que $T f t$ puisse expliquer comment le pansement est si vite arrivé sur mon pouce, sachant que la réciprocité que cette dame peut espérer de quelqu'un qu'elle ne reverra jamais égale zéro.

\section{Simulation, trahison}

Je peux entendre certaines réactions, motivées par le caractère incroyablement simpliste de ces jeux que l'on fait exécuter aux machines. La réalité humaine est infiniment plus compliquée, plus variée, plus belle. Les êtres humains sont intelligents, ils sont capables d'apprécier les situations et de décider pour chacune d'elles une conduite proportionnée à tenir. Rien à voir, donc, avec ces jeux stériles que l'on fait tourner sur les ordinateurs. Personne ne songe à ramener l'art culinaire français à une interaction systématique entre deux ingrédients. La coopération est

Jean-Louis Dessalles, "Coalition Factor in the Evolution of Non-Kin Altruism ", Advances in Complex Systems, vol. 2, no 2, 1999, p. 143-172 [en ligne] http://www.dessalles.fr/papiers/pap.evol/Dessalles_99091402.pdf. 
une conséquence de notre intelligence qui nous permet d'adopter des conduites conjointes lorsque nous anticipons qu'elles se révéleront profitables.

Cette manière de se draper dans la complexité pour maintenir un certain flou théorique me gêne profondément. Certes, les simulations sont simplistes et incroyablement réductrices. Mais elles le sont seulement si on les compare aux "vrais " humains ou aux « vraies » sociétés. Or, il ne s'agit pas de cela. La simulation n’a jamais pour but d'offrir une image réaliste d'un phénomène. Sinon, comme l'a dit Douglas Hofstadter, la simulation d'un ouragan devrait mouiller l'ordinateur. L'objectif d'une simulation est de reproduire fidèlement, non le phénomène lui-même, mais les modèles qui prétendent l'expliquer. Et là, pas de soupçon de réductionnisme, car les modèles imaginés par les scientifiques, quelle que soit leur discipline, sont déjà simplistes. C'est d'ailleurs là une marque de leur qualité. Imaginez une raison qui pousse les humains à coopérer. Formulez-la en un modèle clair et concis. C'est ce modèle qui sera mis à l'épreuve dans la simulation. Et, ô surprise, bien des modèles pourtant évidents et allant de soi ne passent pas l'épreuve et se révèlent erronés.

L'époque que nous vivons est extraordinaire à cet égard : l'ordinateur apporte à de nombreuses disciplines autrefois coupées de toute idée d'expérimentation les moyens de faire jeu égal avec les sciences dites dures. La simulation nous tend un tube à essai. Nous avons le choix entre le regarder comme un objet étrange, réservé au chimiste, ou comme un contenant utile prêt à recevoir ce que nous voudrons bien y mettre.

Certes, mais la réduction persiste dans le fait que la simulation prétend expliquer des phénomènes macroscopiques à l'aide d'interactions locales. Le contentieux est lourd dans les sciences sociales, notamment depuis Durkheim : «La cause déterminante d'un fait social doit être cherchée parmi les faits sociaux antécédents, et non parmi les états de la conscience individuelle ${ }^{3}$ ". La

3 Émile Durkheim, Les règles de la méthode sociologique, Paris, PUF, 1967 [1894], p. 124 [en ligne] http://classiques.uqac.ca/classiques/Durkheim_ emile/regles_methode/durkheim_regles_methode.pdf. 
querelle de l'autonomie du niveau macroscopique est bien connue en économie, où l'on accuse les micro-économistes de réaliser de superbes modèles tout à fait cohérents et calculables, mais découplés de la réalité. Je crois que la Science n'a pas besoin de ce type de querelle. Si vous pensez qu'un phénomène macroscopique ne peut s'expliquer qu'à l'aide de paramètres purement macroscopiques, très bien, tentez votre chance. Mais n'empêchez pas celle ou celui qui croit pouvoir expliquer certaines données comme émergentes, de travailler. Souvenons-nous que ce genre de querelle a existé dans les sciences physiques (elles ont été fatales au grand physicien Ludwig Boltzmann), où personne ne songe à les ressusciter.

\section{Le langage n'est pas une coopération}

Faisons un petit détour par le langage. La communication humaine est fréquemment considérée comme une forme de coopération : on voit que les individus se donnent mutuellement des informations, et qu'ils alternent dans ce rôle. Selon certaines théories, les locuteurs vont même jusqu'à calculer leur message de manière à minimiser le travail d'interprétation de l'auditeur. Si ce n'est pas par pure gentillesse, ce doit être de la coopération. Pas étonnant, puisque l'être humain est censé être, par nature, coopératif.

Ce type de constat résulte cependant d'un examen pour le moins superficiel des données du langage. Examinons un compterendu plus réaliste.

[Cooperation] does not describe the human species as I know it. Watch any group of people conversing, and you will see the exact opposite of the behaviour predicted by the kinship and reciprocity theories of language. People compete to say things. They strive to be heard. When they appear to be listening, they are often mentally rehearsing their next contribution to the discourse rather than absorbing what was just said by others. Those who fail to yield the floor to their colleagues are considered selfish, not altruistic. Turn-taking rules have emerged to regulate not who gets to listen, but who gets to talk. Scientists compete for the chance to give talks at conferences, not for the chance to listen.

$\overline{4}$ Geoffrey F. Miller, The Mating Mind, New York, Doubleday, 2000, p. 350. 
Si le langage n'est pas une coopération, quel est l'avantage des locuteurs? Pourquoi donner ainsi gratuitement des informations potentiellement intéressantes à qui veut bien les entendre, et pire, pourquoi entrer en compétition pour cela?

Au milieu des années 1990, j’ai défendu l'idée que la conversation, loin d'être une coopération, était une compétition pour paraître pertinent ${ }^{5}$. Que gagne-t-on dans cette compétition? Ma réponse de l'époque était : du statut social. L'idée me semblait limpide, inattaquable. On gagne à parler de manière pertinente, car cela permet d'augmenter son existence sociale. Et les auditeurs ont intérêt à donner du statut aux locuteurs pertinents, car... Il me fallait combler ces trois points de suspension. Je me suis tourné vers la simulation, qui ne m’a renvoyé, pendant plusieurs années, que des résultats négatifs. Toutes mes intuitions (introduire des " points de statut ", notamment) produisaient des situations instables, équivalentes à celle de la coopération.

J'ai finalement réussi, après plusieurs années, à produire un modèle, plausible à mes yeux, qui passe le test de la simulation. Il m'a fallu renoncer à l'idée que le statut social entrait directement dans l'équation de base. Une communication de type humain peut émerger si c'est un moyen, tant pour le locuteur que pour l'auditeur, de constituer un réseau social efficace. Le modèle produit une communication stable dès lors que les individus trouvent avantage à avoir des amis capables d'être pertinents (toutes choses égales par ailleurs). Ainsi, le locuteur parle pour montrer sa pertinence, car c'est un moyen d'attirer des amis. L'auditeur écoute et jauge, car c'est pour lui le moyen de ne pas se tromper d'ami. Lidée est simple, et la simulation montre qu'elle est cohérente.

Reste à expliquer la clé de voûte du modèle : pourquoi est-il avantageux, si l'on doit choisir entre deux amis potentiels, d'opter pour le plus pertinent des deux? L'explication que je

5 Jean-Louis Dessalles, "Altruism, Status, and the Origin of Relevance ", dans James R. Hurford, Michael Studdert-Kennedy et Chris Knight (dir.), Approaches to the Evolution of Language: Social and Cognitive Bases, Cambridge, Cambridge University Press, 1998, p. 130-147 [en ligne] http://www.dessalles.fr/papiers/pap.evol/Dessalles_96122602.pdf. 
propose est de nature anthropologique, voire éthologique. Certains auteurs ont noté qu'une différence essentielle entre notre espèce et d'autres espèces de primates est la facilité du meurtre par surprise, rendu possible par l'emploi d'une arme (à commencer par un simple caillou) ${ }^{6}$. Ce changement a bouleversé l'organisation sociale de notre espèce. L'information a remplacé la force physique comme atout essentiel dans les coalitions, car elle seule permet de prévenir un danger qui peut venir à tout moment de votre voisin.

Le résultat est connu : nous prononçons quotidiennement quelque 15000 mots $^{7}$ durant un tiers de notre temps éveille ${ }^{8}$ pour commenter des événements ou des faits inattendus. Ceux qui ne le font pas n'attirent pas d'amis et se retrouvent seuls. Or, dans le contexte naturel de notre espèce, ceux qui n'ont pas d'amis sont les victimes désignées de ceux qui en ont.

\section{Peut-on se passer du concept de coopération?}

Ce qui précède décrit un modèle non coopératif de la communication humaine. Peut-on l'étendre et tenter de faire l'économie de la coopération dans l'étude d'autres conduites sociales? Ce travail reste à faire. La perspective me semble cependant fascinante. À bien y réfléchir, le concept de coopération est pour le moins suspect, tant il ressemble à un cas de wishful thinking. Certains pourront penser que son omniprésence dans l'explication théorique des sociétés humaines par les sciences sociales tient lieu d'une sorte de mythe, porté par une tradition occidentale

$\overline{6}$ James Woodburn, «Egalitarian Societies », Man, no 17, 1982, p. 431-451; Christopher Boehm, Hierarchy in the Forest: The Evolution of Egalitarian Behavior, Harvard (MA), Harvard University Press, 2000; Paul M. Bingham, "Human Evolution and Human History: A Complete Theory ", Evolutionary Anthropology, vol. 9, $\mathrm{n}^{\circ}$ 6, 2001, p. 248-257.

7 Matthias R. Mehl, Simone Vazire, Nairan Ramirez-Esparza, Richard B. Slatcher et James W. Pennebaker, "Are Women Really More Talkative Than Men? ", Science, vol. 317, no 5834, 2007, p. 82.

8 Matthias R. Mehl et James W. Pennebaker, "The Sounds of Social Life: A Psychometric Analysis of Students' Daily Social Environments and Natural Conversations ", Journal of Personality and Social Psychology, vol. 84, $\mathrm{n}^{\circ} 4$, 2003, p. 857-870. 
d'inspiration religieuse, prônant la gentillesse (voire l'amour!) envers le voisin. Il existe peut-être un argument de portée plus générale.

Reprenons la question clé de la sociologie des primates en général, et humaine en particulier : sur quels critère vais-je choisir mes amis? La pertinence est sans doute l'un de ces critères, ce qui a pu provoquer, nous l'avons vu, l'émergence du langage?'. Ce n'est pas le seul, et il sera passionnant de dresser un inventaire des critères d'affiliation, en le soumettant chaque fois à l'épreuve de la simulation. Je crois que la volonté de coopérer est l'un de ces critères. Toutes choses égales par ailleurs, il est préférable de choisir un ami prêt à coopérer plutôt qu'un individu aux tendances égoïstes. Attention : il ne s'agit pas ici de la coopération effective, celle qui est coûteuse et encourage la tricherie. Il s'agit de son affichage. Un tel modèle prédit, non que les individus vont coopérer, mais qu'ils vont tout faire pour démontrer qu'ils sont prêts à coopérer. Voilà qui cadre mieux, on en conviendra, avec la réalité humaine. Dans cette approche des conduites sociales humaines, le comportement n'est pas un ensemble d'actes motivés par un espoir de rentabilité tangible. Chaque acte est un signal. Chaque action visible par autrui est destinée à attirer ou à conserver des amis. La personne qui a si gentiment appliqué un pansement sur mon doigt blessé ne l'a pas fait pour qu'à mon tour, un jour, je lui porte secours dans des conditions analogues. Son acte était un signal, à mon intention, bien sûr, mais également à l'intention de ses amies présentes : "Regardez, je suis capable d'aider quelqu'un que je ne connais pas ". Le spectateur ne s'y trompe pas : nous trouvons ces actes plus " nobles » en tant que signaux, car ils n'attendent pas de rétribution de la part de leur bénéficiaire. Et le réflexe ne se fait pas attendre : nous avons un élan de sympathie, qui révèle que nous descendons d'individus qui ont choisi leurs amis selon de tels critères. Ces signaux en forme d'actes généreux ressemblent

$9 \quad$ Jean-Louis Dessalles, Aux origines du langage : une histoire naturelle de la parole, Paris, Hermès-science, 2000 [en ligne] http://www.dessalles.fr/papiers/ pap.evol/Dessalles_99111703.html. 
métaphoriquement à une coopération, mais n'en sont pas. Ne nous laissons pas abuser par une ressemblance superficielle.

\section{Bibliographie}

Axelrod, Robert, The Evolution of Cooperation, New York, Basics Books, 1984.

Axelrod, Robert et William D. Hamilton, "The Evolution of Cooperation ", Science, vol. 211, no 4489, 1981, p. 1390-1396 [en ligne] http://www. cdnresearch.net/pubs/others/axelrod_ham_1981.pdf.

Bingham, Paul M., "Human Evolution and Human History: A Complete Theory ", Evolutionary Anthropology, vol. 9, no 6, 2001, p. 248-257.

Boehm, Christopher, Hierarchy in the Forest: The Evolution of Egalitarian Behavior, Harvard (MA), Harvard University Press, 2000.

Dessalles, Jean-Louis, Aux origines du langage : une histoire naturelle de la parole, Paris, Hermès-science, 2000 [en ligne] http://www.dessalles.fr/ papiers/pap.evol/Dessalles_99111703.html.

Dessalles, Jean-Louis, "Altruism, Status, and the Origin of Relevance ", dans James R. Hurford, Michael Studdert-Kennedy et Chris Knight (dir.), Approaches to the Evolution of Language: Social and Cognitive Bases, Cambridge (MA), Cambridge University Press, 1998, p. $130-$ 147 [en ligne] http://www.dessalles.fr/papiers/pap.evol/Dessalles_96122602. pdf.

Dessalles, Jean-Louis, "Coalition Factor in the Evolution of Non-Kin Altruism ", Advances in Complex Systems, vol. 2, n 2, 1999, p. 143-172 [en ligne] http://www.dessalles.fr/papiers/pap.evol/Dessalles_99091402. pdf.

Durkheim, Émile, Les règles de la méthode sociologique, Paris, PUF, 1967 [1894], p. 124 [en ligne] http://classiques.uqac.ca/classiques/ Durkheim_emile/regles_methode/durkheim_regles_methode.pdf.

Mehl, Matthias R. et James W. Pennebaker, "The Sounds of Social Life: A Psychometric Analysis of Students' Daily Social Environments and Natural Conversations ", Journal of Personality and Social Psychology, vol. 84, n 4, 2003, p. 857-870. 
Mehl, Matthias R., Simone Vazire, Nairan Ramirez-Esparza, Richard B. Slatcher et James W. Pennebaker, «Are Women Really More Talkative than Men? ", Science, vol. 317, n 5834, 2007, p. 82.

Miller, Geoffrey F., The Mating Mind, New York, Doubleday, 2000.

Woodburn, James, "Egalitarian Societies ", Man, n 17, 1982, p. 431451. 